\title{
Numerical Analysis of Notch-Tip Fields in Rubber-Modified Epoxies
}

\author{
A. AL-ABDULJABBAR and J. PAN \\ Mechanical Engineering and Applied Mechanics \\ The University of Michigan \\ Ann Arbor, Michigan 48109
}

\begin{abstract}
In this work, the near-tip fields in notched specimens of pressure-sensitive nonporous and porous materials are investigated by finite element analysis. The specimen geometry and material properties are adopted from the corresponding experiments on rubber-modified epoxies. The Drucker-Prager yield criterion is first used to describe the yielding of nonporous materials. The yielding behavior of porous materials is based on a generalized Gurson yield criterion. The yield criterion for porous materials accounts for both the matrix material pressure sensitivity and the macroscopic pressure sensitivity due to porosity. Modifications are made on the yield criterion under negative mean stresses in order to account for the specific loading and geometry of the specimen. The computational results are compared with observed experimental cavitation zones and intense shear zones near the notch tip in specimens. Moreover, the near-tip fields and crack initiation sites ahead of the notch tip related to the volume fraction of rubber particles are investigated. The computational results suggest that the lowering of the mean stress ahead of the tip in rubber-modified epoxies with higher volume fractions of rubber changes the fracture mode from being controlled by high mean stresses at the elastic-plastic boundary to being controlled by large plastic strains closer to the notch tip.
\end{abstract}

\section{INTRODUCTION}

$\mathrm{T}$ he yielding of many materials, including polymeric materials, exhibits dependence on the hydrostatic stress, for example, see (1-4). This dependence on the hydrostatic stress is accounted for, from the macroscopic phenomenological viewpoint, by modifying the von Mises yield criterion through the introduction of the mean stress in the yield criterion for pressure-insensitive materials, for example, see Drucker and Prager (5) and Drucker (6).

Modifying thermoset epoxy resins with rubber particles is known to noticeably improve the fracture toughness of the epoxies as shown by McGarry and co-workers (7-9). The toughening by rubber particles increases the range of applicability of plastics. Yee and Pearson (10) and Pearson and Yee $(11,12)$ studied experimentally the fracture behavior of rubber-modified epoxies and pointed out that the mechanism responsible for the increased toughness of rubber-modified epoxies, when compared to pure epoxies, is the cavitation in rubber particles early in the deformation process, followed by the intense shear yielding of the surrounding matrix material.

Based on the experimental work on notched specimens of rubber-modified epoxies, Yee et al. (13) sug- gested that the cavitation of the rubber particles around the notch tip induces a plane-stress condition in the region ahead of the notch tip and thus relieves the thickness constraint even in thick specimens where the plane-strain condition is satisfied. They also noted that the intense shear yielding zone, which is contained within a larger cavitation zone, extends directly ahead of the notch tip.

Ductile fracture mechanisms in metals have been modeled extensively (14). In general, the fracture of ductile metals is intimately related to second-phase particles which experience fracture or decohesion during loading. This leads to the creation of voids and cracks within metals. The presence of such microvoids and cracks gives rise to noticeable macroscopic pressure-sensitive yielding behavior even when the yielding behavior of metals is not significantly pressure dependent. Ductile fracture in metals could occur through a process of nucleation of microvoids, subsequent growth and coalescence of microvoids to final fracture (14).

Gurson $(15,16)$ developed a macroscopic pressuresensitive yield criterion to describe the yielding behavior of porous materials with pressure-insensitive von Mises matrices. Jeong (17) and Jeong and Pan (18, 19) analyzed porous materials with pressure-sensitive 
matrices and proposed a generalized Gurson's yield criterion so that both the microscopic pressure sensitivity in pressure-sensitive matrices, which is traditionally accounted for by the Drucker-Prager yield criterion, and the pressure sensitivity due to porosity of the materials can be incorporated. They also developed a set of constitutive relations to describe the material rate-dependent constitutive behavior for investigation of the crack-tip fields in rubber-modified epoxies. As recently shown by Chang and Pan (20), the load-carrying capacities of porous plastics and rubber-modified plastics are quite similar when cavitation in rubber particles occurs early in the deformation history and a failure criterion under large deformation is assumed for the rubber material. This suggests that use of the generalized Gurson's criterion is reasonable to describe the yielding behavior of rubber-modified plastics after cavitation occurs early in rubber particles.

The goal of this work is to investigate the notch-tip fields in specimens of rubber-modified epoxies with different rubber contents used in the work of Yee et al. (13) based on the generalized Gurson yield criterion by a finite element analysis. A comparison of the computational results and the experimental results is used to assess the proposed mechanisms of toughening and failure in Yee et al. (13). In this paper, a finite element model is first developed for double-edge double-notch specimens used in the experimental work of Yee et al (13). Some preliminary analyses are conducted in order to confirm the accuracy and stability of the finite element model and to assess the possibility of using the Drucker-Prager yield criterion to investigate the crack-tip fields in rubber-modified epoxies. The results from these finite element analyses are compared with the fully-plastic analytical notch-tip stress solutions developed by Al-Abduljabbar and Pan (21). The analytical solutions were developed for wedge-shape circular notch tips with different wedge angles. The analytical solutions include the notch-tip geometry in the specimen used in the experiment of Yee et al. (13). By benchmarking the computational results with the fully plastic analytical stress solutions, we can assess the accuracy of the finite element analysis. Since the fully plastic solutions are applicable to perfectly plastic materials, a perfectly plastic material behavior is assumed in the finite element code ABAQUS with material softening and directional hardening being neglected.

Then, the notch-tip fields in specimens of rubbermodified epoxies are investigated by using the constitutive relations developed by Jeong and Pan (18). Due to the geometry and loading conditions, the yield criterion used in the analysis of rubber-modified epoxies has to cover both positive and negative mean stress states. The region under negative mean stress is described by the Drucker-Prager yield criterion with consideration of the effects of rubber particles on the yield criterion, whereas the region of positive mean stress is described by the generalized Gurson yield criterion proposed by Jeong and Pan (18). The notchtip fields in the specimens are analyzed for different rubber contents, and the results are compared with the experiment of Yee et al. (13).

\section{TIELD CRITERION}

The Drucker-Prager yield criterion has been widely used to account for the effects of the hydrostatic pressure on yielding by a combination of the effective stress $\sigma_{e}$ and the mean stress $\sigma_{m}$ as

$$
\Phi_{D P}(\boldsymbol{\sigma})=\sigma_{e}+\sqrt{3} \mu \sigma_{m}=\sigma_{0}
$$

where $\Phi_{D P}$ represents the yield criterion, $\sigma$ is the Cauchy stress tensor, $\mu$ represents the pressure sensitivity, and $\sigma_{0}$ represents the generalized effective tensile yield stress. Here, $\sigma_{0}$ equals a constant for perfectly plastic materials. The mean stress $\sigma_{m}$ and the effective tensile stress $\sigma_{e}$ are defined as follows

$$
\begin{gathered}
\sigma_{m}=\frac{1}{3} \sigma_{k k} \\
\sigma_{e}=\left(\frac{3}{2} s_{i j} s_{i j}\right)^{1 / 2} .
\end{gathered}
$$

In Eq $3, s_{i j}$ are the deviatoric stress components defined as

$$
s_{i j}=\sigma_{i j}-\sigma_{m} \delta_{i j},
$$

where $\delta_{i j}$ is the Kronecker delta. The subscripts $i, j$ and $k$ range from 1 to 3 and the summation convention for repeated indices is adopted.

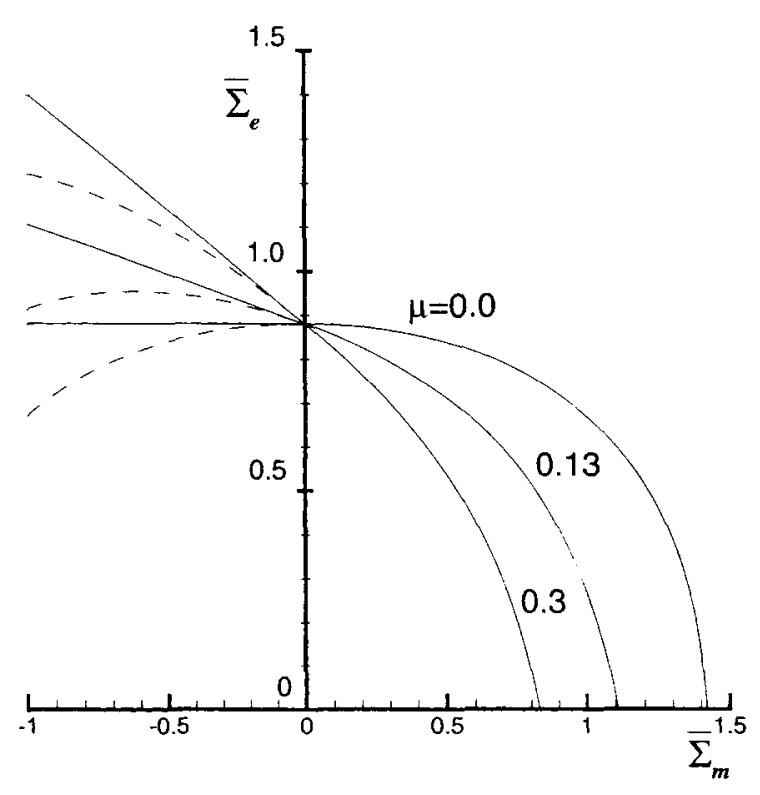

Fig. 1. Yield contours for pressure-sensitive porous materials. In the negative mean stress region, the solid lines represent Eq 7 and the dotted lines represent Eq 6. 


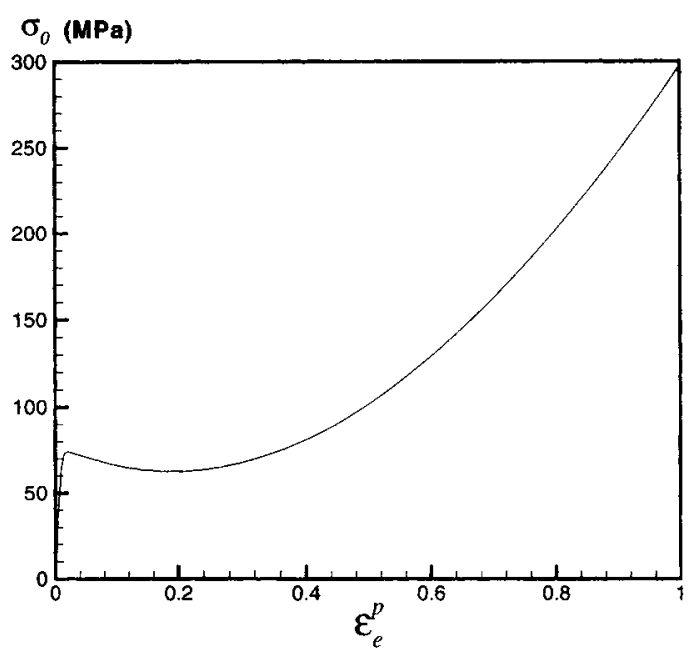

Fig. 2. The stress-strain relation with strain softening-hardening described in Eq 16.

For maraging and tempered martensitic steels, the pressure sensitivity $\mu$ is small with a range of 0.014 to 0.064 as reported in (22-24). For zirconia-containing ceramics, the pressure sensitivity $\mu$ for phase transformation ranges between 0.55 and 0.77 as shown in (25). For polymers, Kinloch and Young (4) reported that $\mu$ ranges from 0.10 to 0.25 . When $\mu$ becomes zero, the Drucker-Prager yield criterion reduces to the von Mises yield criterion. Many more references on using the Drucker-Prager yield criterion to describe the yielding behavior of plastics can be found in Jeong and Pan (18).

The presence of voids and microdefects in materials gives rise to noticeable dependence of yielding on pressure even when the yielding behavior of the matrix materials is not pressure dependent. Based on an upper bound analysis, Gurson (15) presented a pressure dependent yield criterion for porous materials. Tvergaard $(26,27)$ modified Gurson's yield criterion by three parameters $q_{1}, q_{2}$, and $q_{3}$ to fit the results of his finite element analysis for localization as

$$
\begin{aligned}
\Phi\left(\Sigma, \sigma_{0}, f\right)=\left(\frac{\Sigma_{e}}{\sigma_{0}}\right)^{2} & +2 f q_{1} \cosh \left(q_{2} \frac{3 \Sigma_{m}}{2 \sigma_{0}}\right) \\
& -1-q_{3} f^{2}=0,
\end{aligned}
$$

where $\Phi$ represents the yield criterion, $\Sigma$ is the macroscopic Cauchy stress tensor acting on the porous material, and $f$ is the void volume fraction. The macroscopic effective tensile stress $\Sigma_{e}$ and the macroscopic mean stress $\Sigma_{m}$ are defined in a fashion similar to that of $\sigma_{e}$ and $\sigma_{m}$ above. Jeong and Pan (18) generalized this form in order to include the effect of the pressure sensitivity of the matrix materials as follows

$$
\begin{aligned}
\Phi\left(\Sigma, \sigma_{0} f . \mu\right) & =\left(\frac{\Sigma_{e}+\mu^{*} \Sigma_{m}}{\sigma_{0}}\right)^{2}+2_{q 1} f \cosh \\
& \left(q_{2} \frac{3+\mu^{*}}{2 \mu^{*}} \log \left(1-\mu^{*} \frac{\Sigma_{m}}{\sigma_{0}}\right)\right)-1-q_{3} f^{2}=0
\end{aligned}
$$

where $\mu^{*}=\sqrt{3} \mu$. Here, the parameters $q_{1}, q_{2}$, and $q_{3}$ are also introduced to fit the yield criterion to the corresponding finite element results. The values of these parameters are different from those of Tvergaard. In Jeong and Pan (18), the values of these parameters are $q_{1}=1.35, q_{2}=0.95$, and $q_{3}=1.35$. The generalized Gurson criterion given in Eq 6 accounts for the combined effects of the matrix pressure sensitivity and the porosity on the macroscopic pressure-sensitive yielding. When the matrix pressure sensitivity is negligible $(\mu=0), E q 6$ reduces to the Gurson-Tvergaard yield criterion $(E q 5)$. When the porosity is negligible $(f=0), E q$ 6 reduces to the Drucker-Prager yield criterion ( $E q 1)$.

Equation 6 will be used to describe the yielding behavior of the porous material where the mean stress is positive. Yee and Pearson (10-12) noted that the rubber particles were cavitated at very low stresses. As recently shown by Chang and Pan (20), the load-carrying capacity of the rubber-modified plastic, when a rubber failure criterion at large deformation is considered, is nearly the same as that of the corresponding porous plastic. Therefore, the generalized yield criterion for pressure-sensitive porous materials may be used to describe the yielding of rubber-modified epoxies.

In the region where the mean stress is negative, the rubber particles will not be cavitated. Since the rubber is incompressible, a simple rule of mixtures would be the first approximation to depict the yielding behavior with the rubber being considered to contribute to shear load-carrying capacity at small strains. However, due to the consideration of the continuity of the yield criterion near zero mean stress, we neglect the small contribution of rubber to the shear load-carrying capacity when the mean stress is negative and use the following yield criterion

$$
\Phi\left(\Sigma, \sigma_{0}, f, \mu\right)=\left(\frac{\Sigma_{e}+\mu^{*} \Sigma_{m}}{\sigma_{0}}\right)^{2}+2 q_{1} f-1-q_{3} f^{2}=0
$$

Figure 1 shows the yield contours for porous materials with a void volume fraction $f=0.12$ and different values of $\mu$. In the negative mean stress region, the dotted lines represent the yield criterion based on Eq 6 and the solid lines represent the yield criterion based on Eq 7.

Jeong and Pan (18) also proposed a plastic potential function $\Phi_{p}$ similar to the yield criterion, with the pressure sensitivity factor $\mu$ replaced by the dilatancy factor $\beta$. A fictitious generalized tensile flow stress $\sigma_{f}$ is sought such that the current stress state is located at the plastic potential surface in the stress space. The dilatancy of plastics is small; for example, see Spitzig and Richmond (3). Therefore, the dilatancy factor is taken as zero for simplicity. Then the potential function reduces to a form similar to the Gurson-Tvergaard yield criterion and the von Mises yield criterion for positive and negative mean stresses, respectively. 


\section{CONSTITUTIVE RELATIONS}

The constitutive relations for porous materials presented in this section are adopted from the work of Jeong and Pan (18). Although the yield criterion and the plastic potential function discussed above were developed for rate-independent porous materials, we assume that they are approximately applicable to rate-dependent materials (18). The constitutive relations are derived by first decomposing the rate of deformation tensor $\mathbf{D}$ into an elastic and a plastic part

$$
\mathbf{D}=\mathbf{D}^{e}+\mathbf{D}^{p}
$$

The elastic rate of deformation tensor $\mathbf{D}^{e}$ is related to the Jaumann rate of the macroscopic Cauchy stress $\hat{\mathbf{\Sigma}}$ by

$$
\mathbf{D}^{e}=\frac{1}{E^{*}}\left[\left(1+v^{*}\right) \hat{\mathbf{\Sigma}}-v^{*} \mathbf{I}(\mathbf{I}: \hat{\mathbf{\Sigma}})\right]=\mathbf{L}^{-\mathbf{I}}: \hat{\mathbf{\Sigma}}
$$

where $E^{*}$ and $v^{*}$ are the macroscopic elastic modulus and Poisson's ratio of the porous material, respectively. These are defined by a self-consistent model based on the average stress scheme, which was introduced by Tandon and Weng (28)

$$
\begin{aligned}
& E^{*}=\frac{2 E(7-5 v)(1-f)}{14-10 v+f(1+v)(13-15 v)}, \\
& v^{*}=\frac{v(14-10 v)+f(1+v)(3-5 v)}{14-10 v+f(1+v)(13-15 v)}
\end{aligned}
$$

As for the plastic part of the deformation-rate tensor, $D^{p}$ its covariant components are related to the partial derivatives of the plastic potential function $\Phi_{p}$ with respect to the corresponding contravariant components of the macroscopic Cauchy stress tensor $\mathbf{\Sigma}$ as

$$
D_{i j}^{p}=\dot{\Lambda} \frac{\partial \Phi_{p}}{\partial \Sigma^{i j}}
$$

where $\dot{\Lambda}$ is a proportionality factor. The equivalent ten sile plastic strain $E_{e}^{p}$ for porous materials is defined as

$$
E_{e}^{p}=\int_{0}^{t}\left(2 \mathbf{D}^{p^{\prime}}: \mathbf{D}^{p^{\prime}}\right)^{1 / 2} d t,
$$

where $D^{p^{\prime}}$ is the deviatoric part of $D^{p}$. Here, $E_{e}^{p}$ is a convenient scalar quantity for measuring the extent of plastic deformation.

The equivalent plastic work for porous materials can be expressed as in (18):

$$
\mathbf{\Sigma}: \mathbf{D}^{p}=(1-f) \sigma_{p} \dot{\varepsilon}_{e}^{p}
$$

Here, $\dot{\varepsilon}_{e}^{p}$ is the effective plastic strain rate of the matrix materials, which is determined according to

$$
\dot{\varepsilon}_{e}^{p}=\dot{\varepsilon}_{r}\left[\frac{\sigma_{0}}{g\left(\varepsilon_{e}^{p}\right)}\right]^{1 / m},
$$

where $g\left(\varepsilon_{e}^{p}\right)$ is a generalized stress function, $\dot{\varepsilon}_{r}$ is a reference plastic strain rate, and $m$ is the material rate sensitivity. A convenient strain softening-hardening form of $g\left(\varepsilon_{e}^{p}\right)$ is adopted here (18)

$$
g\left(\varepsilon_{e}^{p}\right)=\sigma_{y}\left[\left(1+\bar{\varepsilon}_{e}^{p}\right)^{N}+C_{1}\left(\bar{\varepsilon}_{e}^{p}\right)^{N 1} \log \left(C_{2} \bar{\varepsilon}_{e}^{p}\right)\right],
$$

where $\sigma_{y}=\sqrt{3} \tau_{y}$. Here, $\tau_{y}$ is the yield stress in shear at the reference shear plastic strain rate $\dot{\gamma}_{r}\left(=\sqrt{3} \dot{\varepsilon}_{r}\right)$, and $\bar{\varepsilon}_{e}^{p}=\varepsilon_{e}^{p} /\left(\sigma_{y} / E\right)$. In $E q 16, N$ and $N_{1}$ are the hardening exponents and $C_{1}$ and $C_{2}$ are material coefficients. Figure 2 shows the stress-strain relation based on $g\left(\varepsilon_{e}^{p}\right)$ typical epoxies with $E=2.94 \mathrm{GPa}, \nu=0.4, \sigma_{y}$ $=75 \mathrm{MPa}, m=0.035, N=0.1, N_{1}=1.3, C_{1}=0.03$, and $C_{2}=0.05$ at $\dot{\varepsilon}_{e}^{p}=\dot{\varepsilon}_{r}=0.0032 \mathrm{~s}^{-1}$.

Combining Eqs 12 and 14 gives the relation for $\dot{\Lambda}$ as

$$
\dot{\Lambda}=\frac{(1-f) \sigma_{p} \dot{\varepsilon}_{e}^{p}}{\sum^{i j} \frac{\partial \Phi_{p}}{\partial \Sigma^{i j}}} .
$$

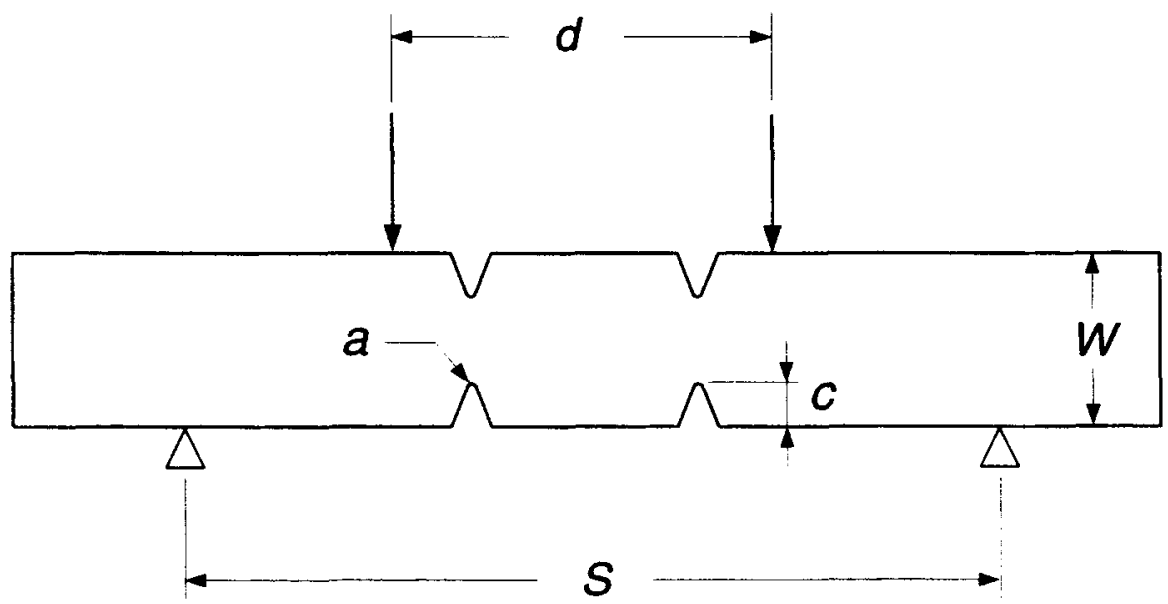

Fig. 3. Geometry and loading of a SDEDN specimen used in Yee et al. (13). 
Fig. 4. (a) The finite element model of the left half of the specimen, (b) The finite element model near the left notch, (c) The coordinate system for the left notch.

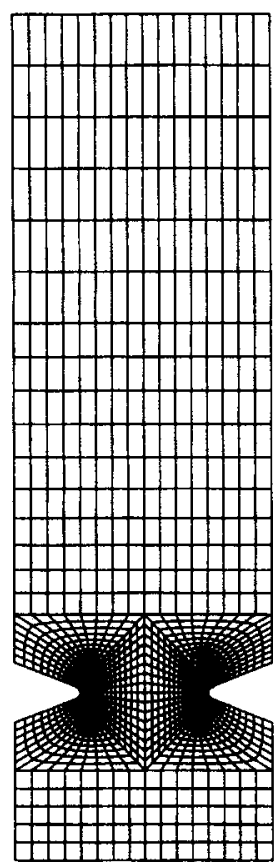

(a)

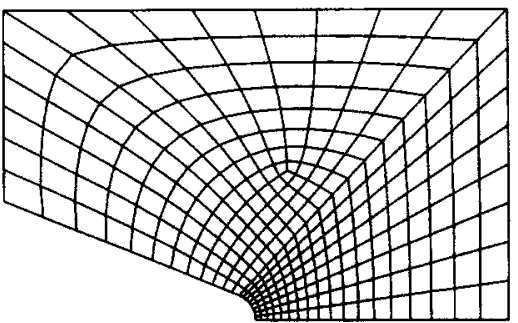

(b)

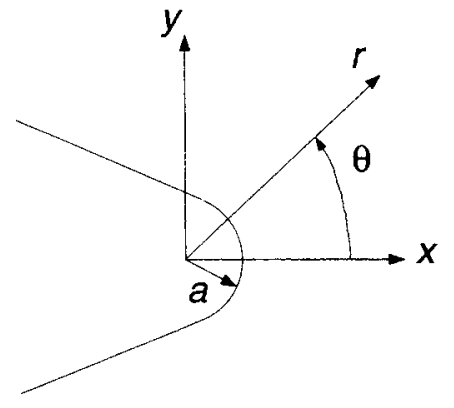

(c)
In addition, the consistency condition which holds during plastic deformation requires that

$$
\dot{\Phi}=\frac{\partial \Phi}{\partial \Sigma^{i j}} \hat{\Sigma}^{i j}+\frac{\partial \Phi}{\partial \sigma_{0}} \dot{\sigma}_{0}+\frac{\partial \Phi}{\partial f} \dot{f}=0,
$$

where the dot denotes the time derivative. In general, the change in the void volume fraction comes from two contributions: growth of the existing voids and nucleation of new voids. In this study, we consider only the growth of the existing voids. Therefore, the rate of the void volume fraction is expressed as

$$
\dot{f}=(1-f) \mathbf{D}^{p}: \mathbf{I}
$$

Note that the reduction in the void growth due to the matrix dilatancy considered in Jeong and Pan (19) is eliminated in Eq 19 because the dilatancy factor $\beta$ is taken as 0 because of the reported small plastic dilatancy of plastics as pointed out earlier.

\section{FINTE ELEMTNT MODEL}

In this section, the pressure-sensitive yield criteria and the constitutive relations for Drucker-Prager materials and for porous materials with Drucker-Prager matrix materials discussed earlier are used in a finite element analysis to investigate the notch-tip fields of symmetrical double-edge double-notched (SDEDN) specimens used in Yee et al. (13). The geometry and loading of a SDEDN specimen are shown in Fig. 3. The specimen has width $(W)$ of $19.1 \mathrm{~mm}$, thickness of $6.3 \mathrm{~mm}$ and length of $126 \mathrm{~mm}$. The notches on each side are $25 \mathrm{~mm}$ apart, and the ratio of notch size to width, $c / W$, is 0.25 . The notches are $V$-shaped with a total wedge angle of $45^{\circ}$ and a notch radius $a=0.50$ $\mathrm{mm}$. The span between the upper load application points, $d$, is $35 \mathrm{~mm}$, and the span between the lower supporting points, $S$, is $75 \mathrm{~mm}$.

Due to the symmetry, only one half of the specimen is modeled in our finite element analysis. The loading is applied on the upper side of the specimen so that the upper portion is under compression, and the lower portion is under tension. The finite element model of the left half of the specimen, rotated $90^{\circ}$ clockwise, is shown in Fig. $4(a)$. The model includes two notches and consists of 1535 elements and 4786 nodes. Figure $4(b)$ shows a close-up of the region near the left notch under tension. Figure 4(c) shows the coordinate systems for the presentation of finite element results. The origins of the polar coordinates and the Cartesian coordinates are at the center of the circular profile of the notch tip. In the finite element analysis, 8-node reduced-integration plane-strain elements are used.

In our finite element analysis, the specimen is subjected to displacement-controlled loading conditions. Prescribed displacement conditions are used at the support and load application points. The displacement at the load application points is increased to the value at which the specimen fractures. From Yee et al. (13), the specimen with 10 p.h.r. CTBN rubber fractures at a total load of $480 \mathrm{~N}$. An equivalent prescribed displacement of the load application point at fracture based on linear elastic beam theory is 1.1 $\mathrm{mm}$. We will use this displacement to study the stress and deformation patterns near the notch tip in the SDEDN specimens. We begin with an analysis of the 
Fig 5. Notch-tip opening stresses in the $y$ direction, normalized by $\sigma_{0}$ directly ahead of the notch tip as functions of $r / a$, for pressuresensitive Drucker-Prager materials with $\mu=0.13$ at different load levels from the FEM analysis. The corresponding fully plastic solution is also shown as the solid line.

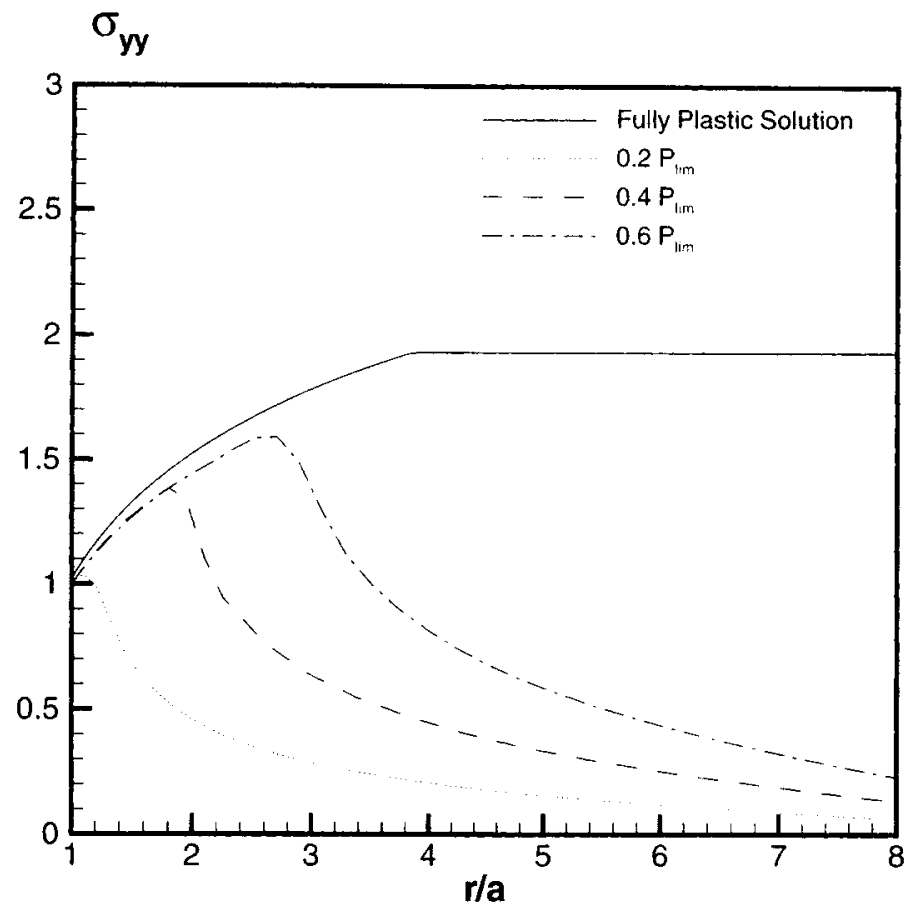

$$
M_{l i m}=\frac{1}{\left(1-\frac{\mu^{2}}{3}\right)^{1 / 2}} \frac{1}{2 \sqrt{3}} \sigma_{0} b^{2}
$$

Drucker-Prager materials. Then, we examine the notchtip fields in porous materials with pressure-sensitive Drucker-Prager matrix materials.

\section{RESULTS FOR DRUCEOR-PRAGER MATERIALS}

For the analyses of the specimen of pressure-sensitive Drucker-Prager materials, the material constants are: $E$ (Young's modulus) $=2.5 \mathrm{GPa}, \nu$ (Poisson's ratio) $=0.4$, and $\sigma_{y}$ (yield stress) $=67 \mathrm{MPa}$, which are typical material properties of epoxies from tensile tests. We only consider elastic perfectly plastic material behavior. We select the elastic-plastic material behavior option in finite element code ABAQUS.

For the convenience of presentation, the stresses presented in this section and next section are normalized by the generalized tensile yield stress $\sigma_{0}$. Figure 5 shows the normalized opening stresses in the $y$ direction, $\bar{\sigma}_{y y}$, as functions of the radial distance normalized by the notch radius, $r / a$, ahead of the notch tip ( $\theta$ $=0$ ), for pressure-sensitive materials with $\mu=0.13$ at different loads from the finite element computations. The normalized fully-plastic solution for the same notch geometry obtained in Al-Abduljabbar and Pan (21) is also presented in the figure as the solid line for $\mu=0.13$. The fully-plastic stress solution is for perfectly plastic materials.

The loads corresponding to the applied displacements are normalized by a lower-bound limit load $P_{\text {lim }}$. The plastic limit load is obtained by assuming full-plastic deformation throughout the remaining ligament of the specimen. A lower-bound limit moment $M_{\text {lim }}$ for the remaining ligament is where $b=W-2 c(30)$. Therefore the corresponding limit load $P_{\text {lim }}$ is obtained as

$$
P_{\text {tim }}=\frac{4 M_{\text {lim }}}{S-d}
$$

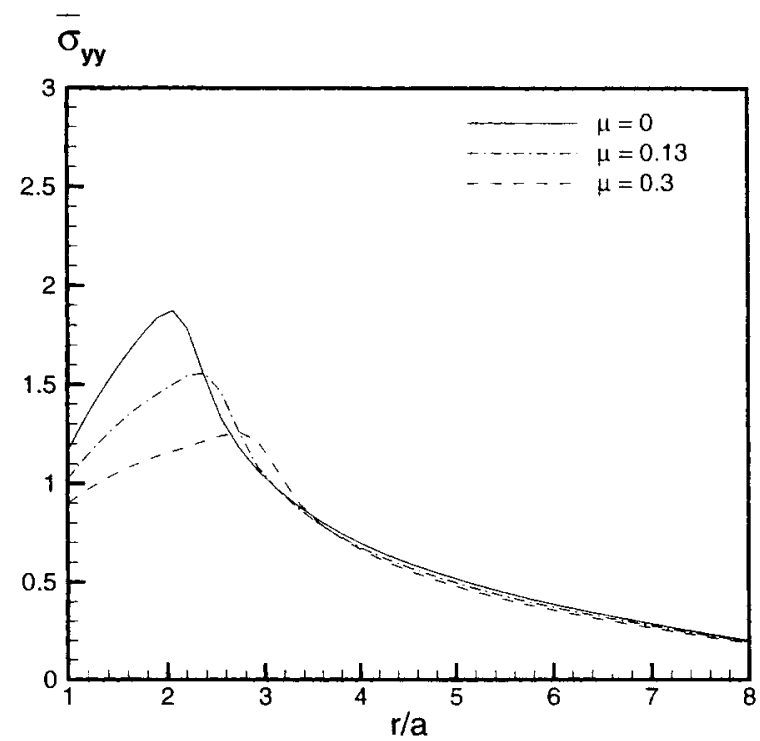

Fig. 6. Notch-tip opening stresses in the $y$ direction, normal ized by $\sigma_{0}$, directly ahead of the notch tip as functions of $r / a$ for von Mises materials with $\mu=O$ and pressure-sensitive Drucker-Prager materials with $\mu=0.13$ and 0.3 at the prescribed displacement of $1.1 \mathrm{~mm}$. 


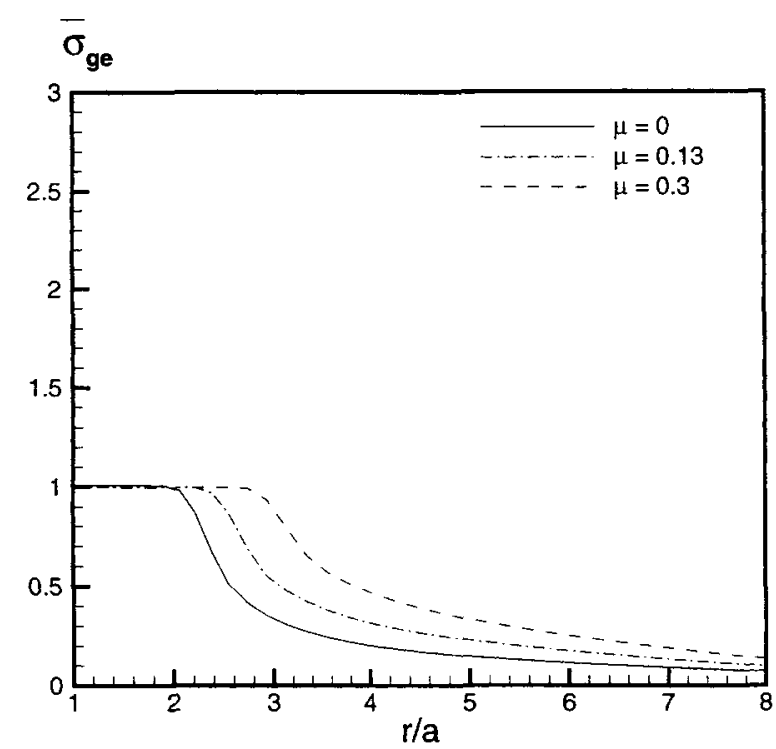

Fig. 7. Generalized tensile effective stresses, normalized by $\sigma_{0}$. directly ahead of the notch tip as functions of $r / a$ for $\mu=$ $0,0.13$, and 0.3 at the prescribed displacement of $1.1 \mathrm{~mm}$.

The details of the derivation for the limit moment are presented in Al-Abduljabbar and Pan (29). In Fig. 5, the results for different loads from the finite element analysis show different stress levels ahead of the notch tip. As the load increases, the finite element results seem to approach to the fully plastic solution for perfectly plastic materials. In contrast to the fully plastic solution, the finite element solutions indicate that beyond the location of the maximum stress, the opening stress decreases as $r$ increases. The finite element results also indicate that the region beyond the maximum stress is in elastic state.

Figure 6 shows the normalized opening stresses, $\bar{\sigma}_{y y}$, ahead of the notch tip as functions of the normalized radial distance $r / a$ for perfectly plastic Mises materials $(\mu=0)$, and two pressure-sensitive Drucker-Prager materials $(\mu=0.13$ and 0.3$)$ at the prescribed displacement of $1.1 \mathrm{~mm}$. For the three cases with $\mu=0$, 0.13 and 0.3 , the uniaxial tensile yield stress is set at $67 \mathrm{MPa}$ for these computations. The plot shows that as the pressure sensitivity $\mu$ increases, the maximum normalized opening stress in the $y$ direction decreases and the extent of the region from the tip to the location of the maximum opening stress increases. The peak value of the stress locates the maximum extent of the plastic region ahead of the notch tip. Beyond the location of the maximum opening stress, the stress decreases as the radial distance from the tip increases. Figure 7 shows the normalized generalized tensile effective stresses, $\sigma_{g e}$, as functions of $r / a$ for $\mu$ $=0,0.13$ and 0.3 at the prescribed displacement of $1.1 \mathrm{~mm}$. When we compare Figs. 6 and 7 , it is clear that in the region beyond the location of the maximum opening stress, the material is in elastic state. It is also clearly shown that the plastic zone size increases as the pressure sensitivity increases at the prescribed displacement.

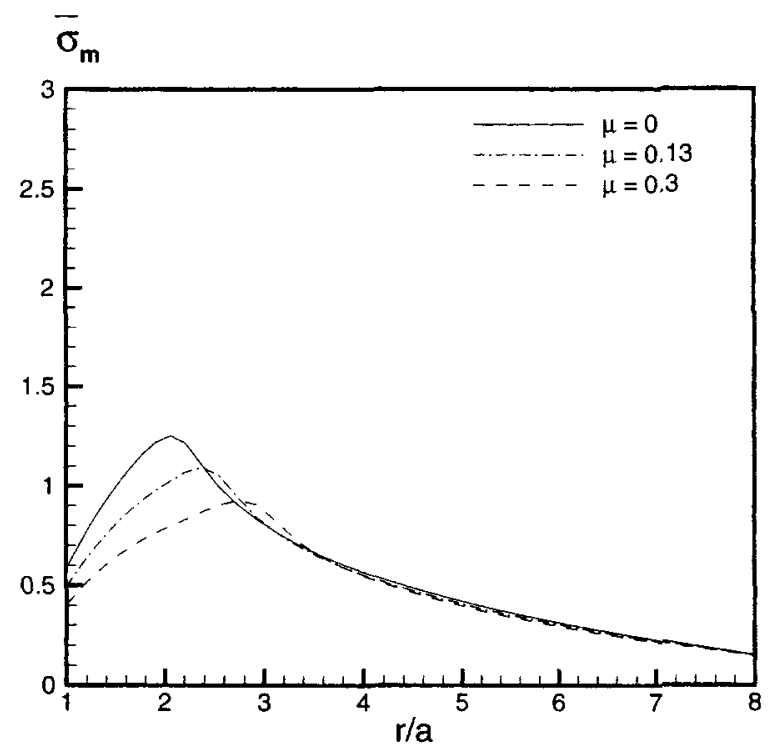

Fig. 8. Mean stresses, normalized by $\sigma_{0}$, directly ahead of the notch tip as functions of $r / a$ for $\mu=0,0.13$ and 0.3 at the prescribed displacement of $1.1 \mathrm{~mm}$.

Figure 8 shows the normalized mean stresses directly ahead of the notch tip for $\mu=0,0.13$ and 0.3 . The trends shown in Fig. 8 are quite similar to those shown in Fig. 6. Fig. 9 shows the equivalent tensile plastic strain $\varepsilon_{e}^{p}\left(=\int_{0}^{t} \sqrt{2 \dot{\varepsilon}_{i j}^{p^{\prime}} \dot{\varepsilon}_{l j}^{p^{\prime}}} d t\right.$, where $\dot{\varepsilon}_{i j}^{p^{\prime}}$ represent the deviatoric plastic strain rates) directly ahead of the notch tip for $\mu=0,0.13$ and 0.3 . As shown in Fig. 9, for higher values of pressure sensitivity, the equivalent tensile plastic strain in the region ahead of the notch tip is larger. The effects of pressure sensitivity on the notch-tip stress fields for pressure-sensitive materials presented in Figs. 5 to 8 are in general agree-

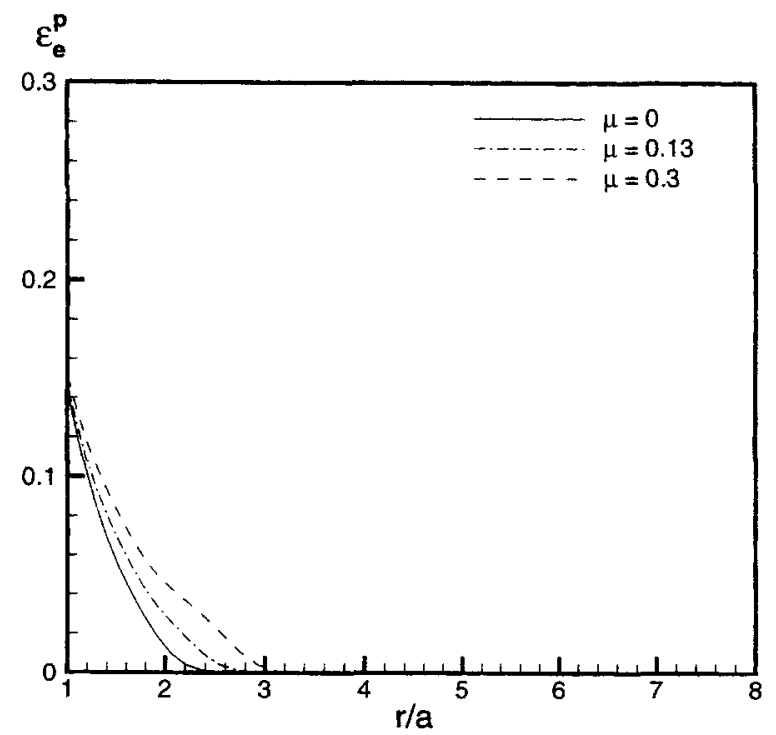

Fig. 9. Equivalent tensile plastic strains directly ahead of the notch tip as functions of $r / a$ for $\mu=0,0.13$, and 0.3 at the prescribed displacement of $1.1 \mathrm{~mm}$. 
$\mu=0$

$\mu=0.13$

Fig. 10. Distributions of the mean stress near the notch tip for $\mu=0$, 0.13 and 0.3 .
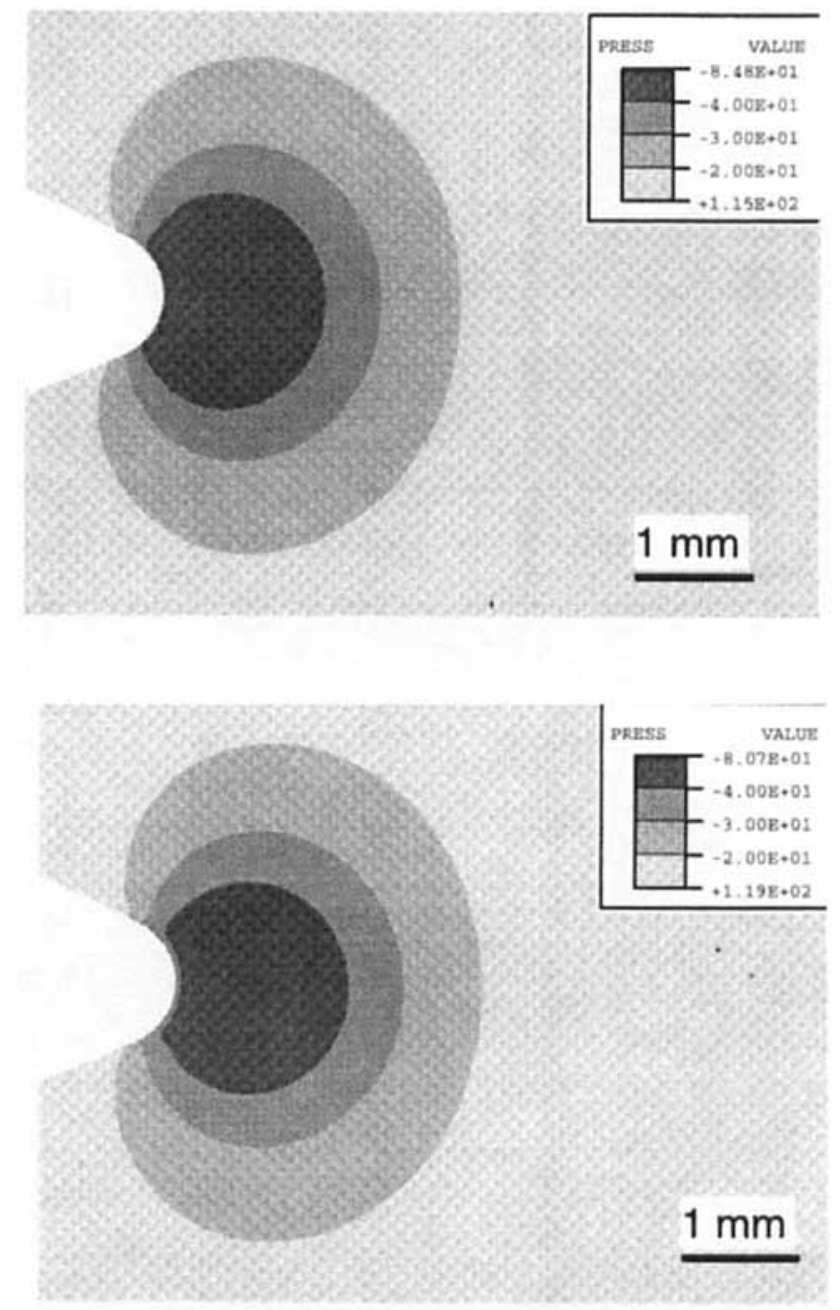

$$
\mu=0.3
$$

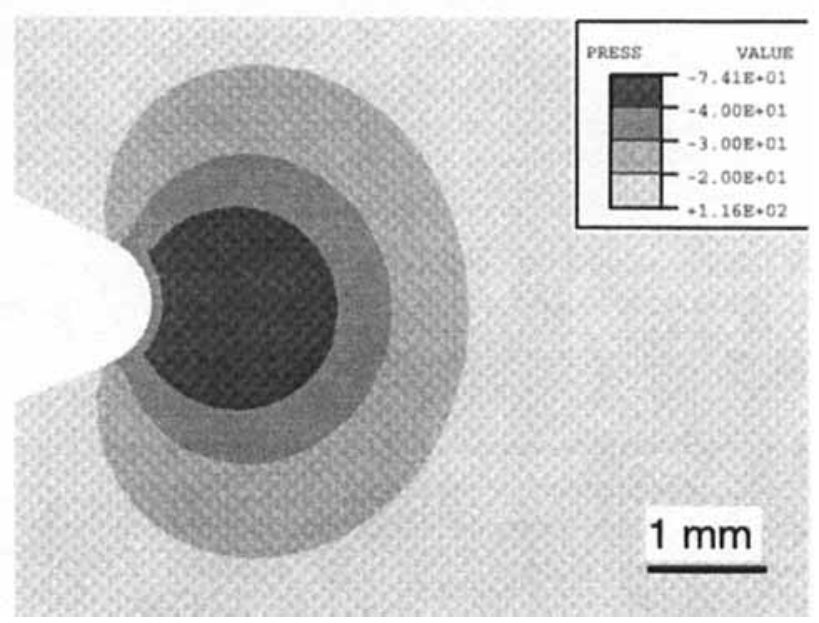

Different mean stress levels corresponding to different hydrostatic pressure levels are plotted.

The change in the shape of the distribution around the notch is quite mild as the pressure sensitivity increases. Figure 11 shows the distributions of the ment with those of the analytical solutions presented in Al-Abduljabbar and Pan (21).

The distributions of the mean stress around the notch tip are shown in Fig. 10 for $\mu=0,0.13$ and 0.3 at the same prescribed displacement of $1.1 \mathrm{~mm}$. 


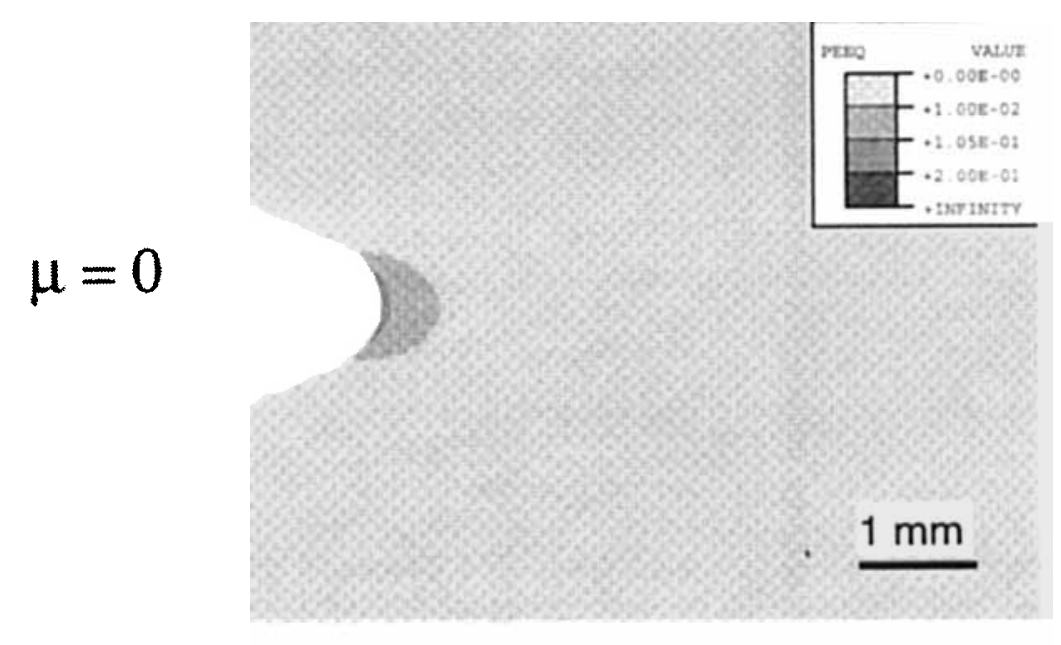

Fig. 11. Distributions of the equivalent tensile plastic strain near the notch tip for $\mu=0,0.13$ and 0.3 .

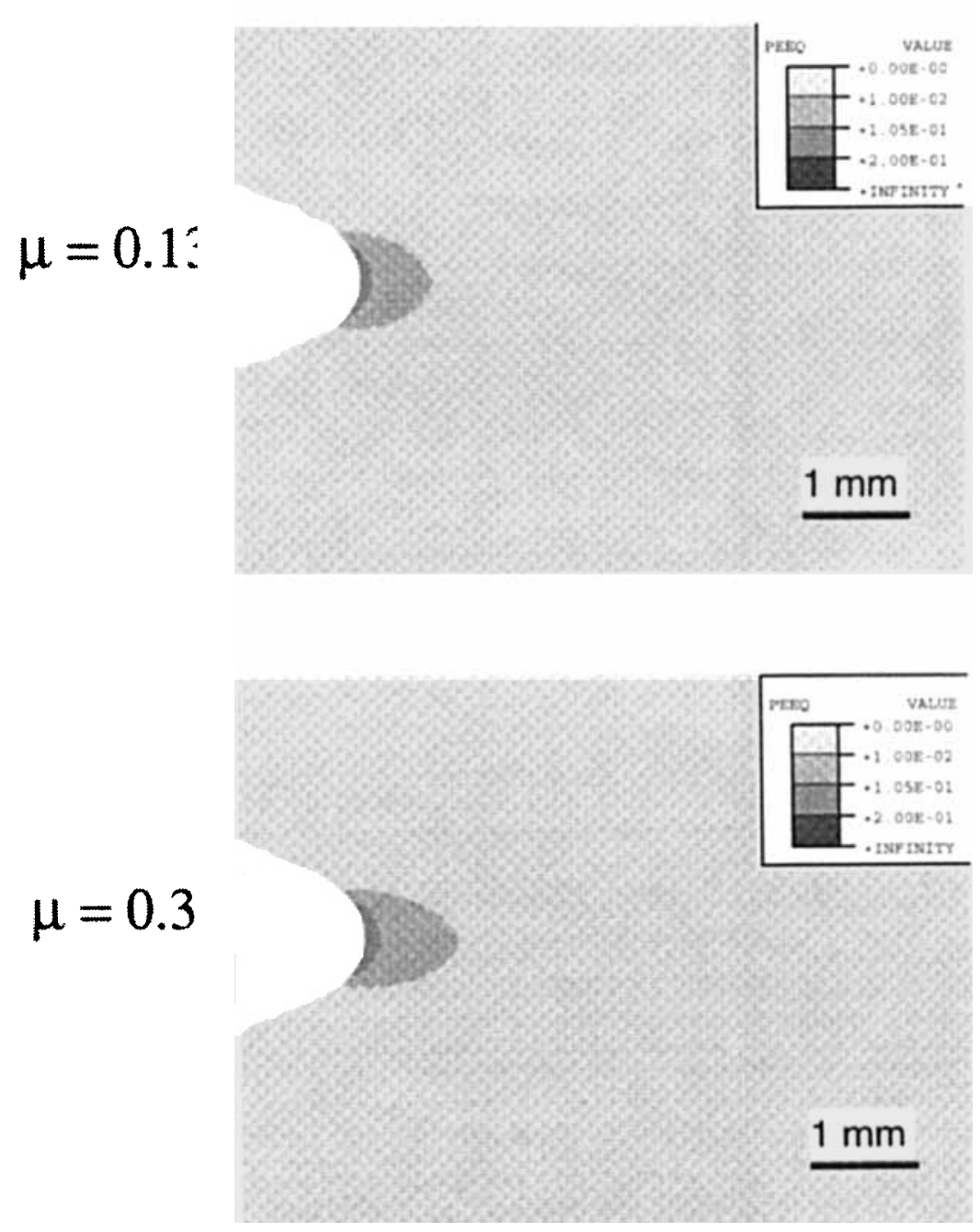

equivalent tensile plastic strain for $\mu=0,0.13$ and 0.3 . The figure shows that as the pressure sensitivity increases, the plastic zone ahead of the notch increases. It should be noted that there is no intense shear zone where the equivalent tensile plastic strains are larger than $\varepsilon_{e}^{p}=0.2$. 


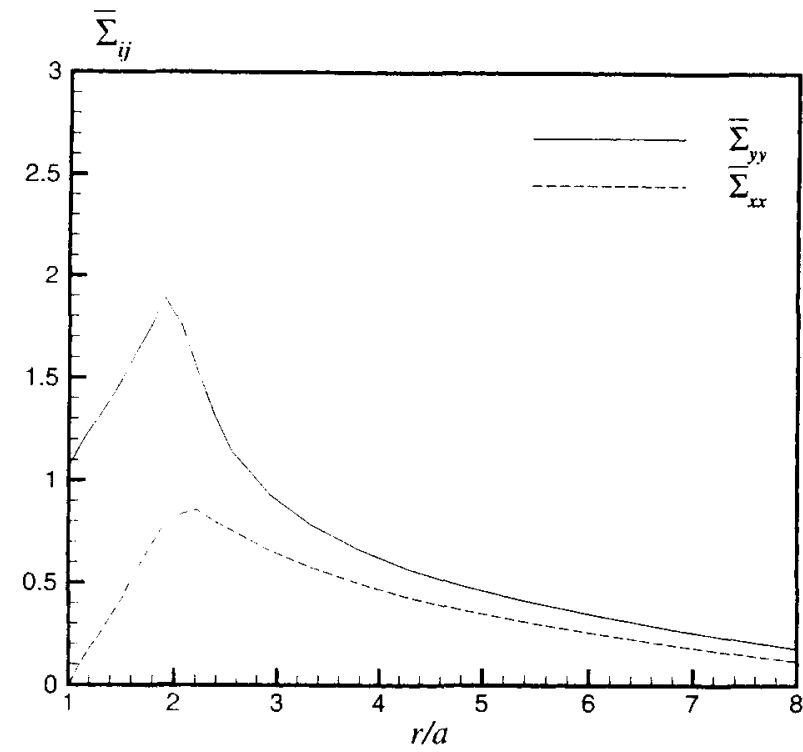

Fig. 12. Macroscopic normal stresses in the $x$ and $y$ directions. normalized by $\sigma_{o}$, directly ahead of the notch tip as functions of $r / a$ for pressure-sensitive porous materials with the softening-hardening behavior, $\mu=0.13$ and $f_{0}=0$.

the notch-tip fields. However, in order to model the notch-tip fields in rubber-modified epoxies, the generalized Gurson's yield criterion proposed by Jeong and Pan (18) is a logical choice since the yield criterion can include the effects of the voids due to cavitation in rubber particles and the yield criterion can be readily used in finite element analyses.

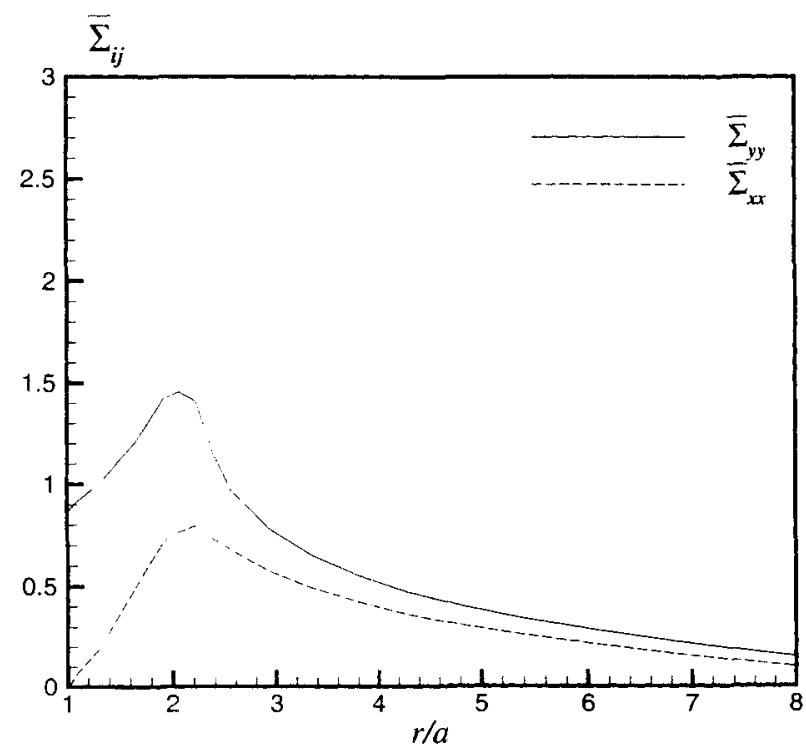

Fig. 13. Macroscopic normal stresses in the $x$ and $y$ directions, normalized by $\sigma_{0}$, directly ahead of the notch tip as functions of $r / a$ for pressure-sensitive porous materials with the softening-hardening behavior, $\mu=0.13$ and $f_{0}=0.06$.

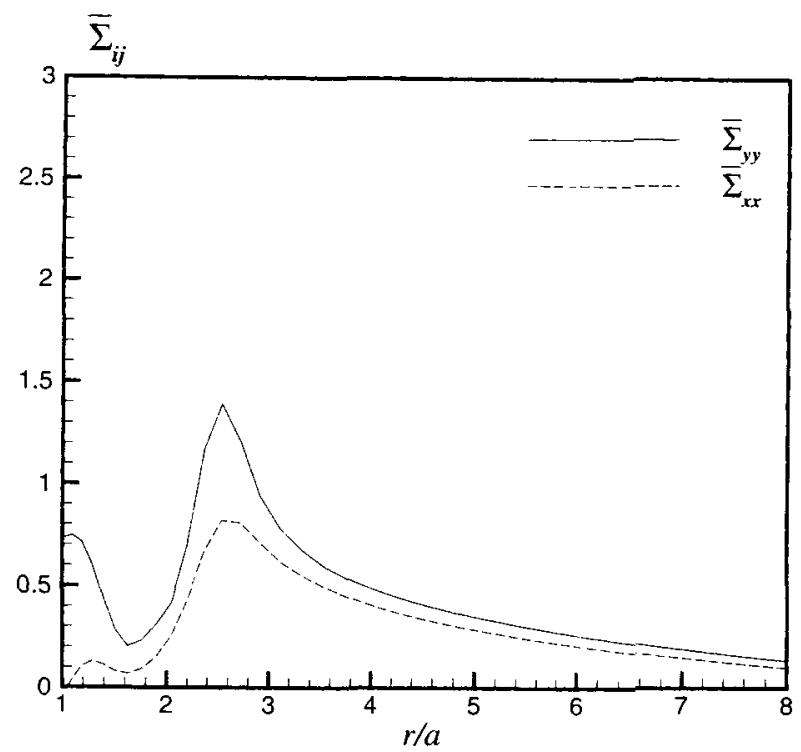

Fig. 14. Macroscopic normal stresses in the $x$ and $y$ directions, normalized by $\sigma_{0}$, directly ahead of the notch tip as functions of $r / a$ for pressure-sensitive porous materials with the softening hardening behavior, $\mu=0.13$ and $f_{0}=0.12$.

\section{RESULTS FOR POROUS MATERULS}

As shown by Chang and Pan (20), the load-carrying capacities of porous plastics and rubber-modified plastics are quite close to each other when cavitation takes place early in the deformation history [at the mean stress of a few MPa, see Gent and Lindley (31)]. Therefore, the use of the constitutive law for porous plastics seems to be appropriate when we investigate the deformation state where the plastics are well into plastic yielding. Therefore, we take the generalized Gurson model of Jeong and Pan for porous materials (18) to describe the macroscopic yielding behavior of rubber-modified epoxies. We take the stress-strain relation as shown in Fig. 2 for the epoxy. All the results in this section are for the same prescribed displacement of $1.1 \mathrm{~mm}$. This prescribed displacement corresponds to the fracture load of the SDEDN specimen as discussed earlier.

The normalized opening and transverse macroscopic stresses $\left(\bar{\Sigma}_{y y}=\Sigma_{y y} / \sigma_{0}\right.$ and $\bar{\Sigma}_{x x}=\Sigma_{x x} / \sigma_{0}$, respectively) ahead of the notch tip are plotted as functions of the normalized radial distance $r / a$ in Figs. 12 to 14 for porous materials with $\mu=0.13$ and the initial void volume fraction of $f_{0}=0,0.06$ and 0.12 , respectively. As shown in Fig. 12 for $f_{0}=0$, the distributions of the stresses are similar to those for Drucker-Prager materials as shown in the previous section but the directional hardening behavior results in a sharper increase to the maximum opening stress at the elasticplastic boundary as the radial distance to the notch tip increases.

As shown in Fig. 13 for the small initial void volume fraction of $f_{0}=0.06$, the magnitudes of the maximum stresses are reduced but without significant change in 
$f_{0}=0$

Fig. 15. Distributions of the macroscopic mean stress near the notch tip for pressure-sensitive porous materials with the softening-hardening behavior, $\mu=0.13$, and $f_{0}=0,0.06$ and 0.12 .
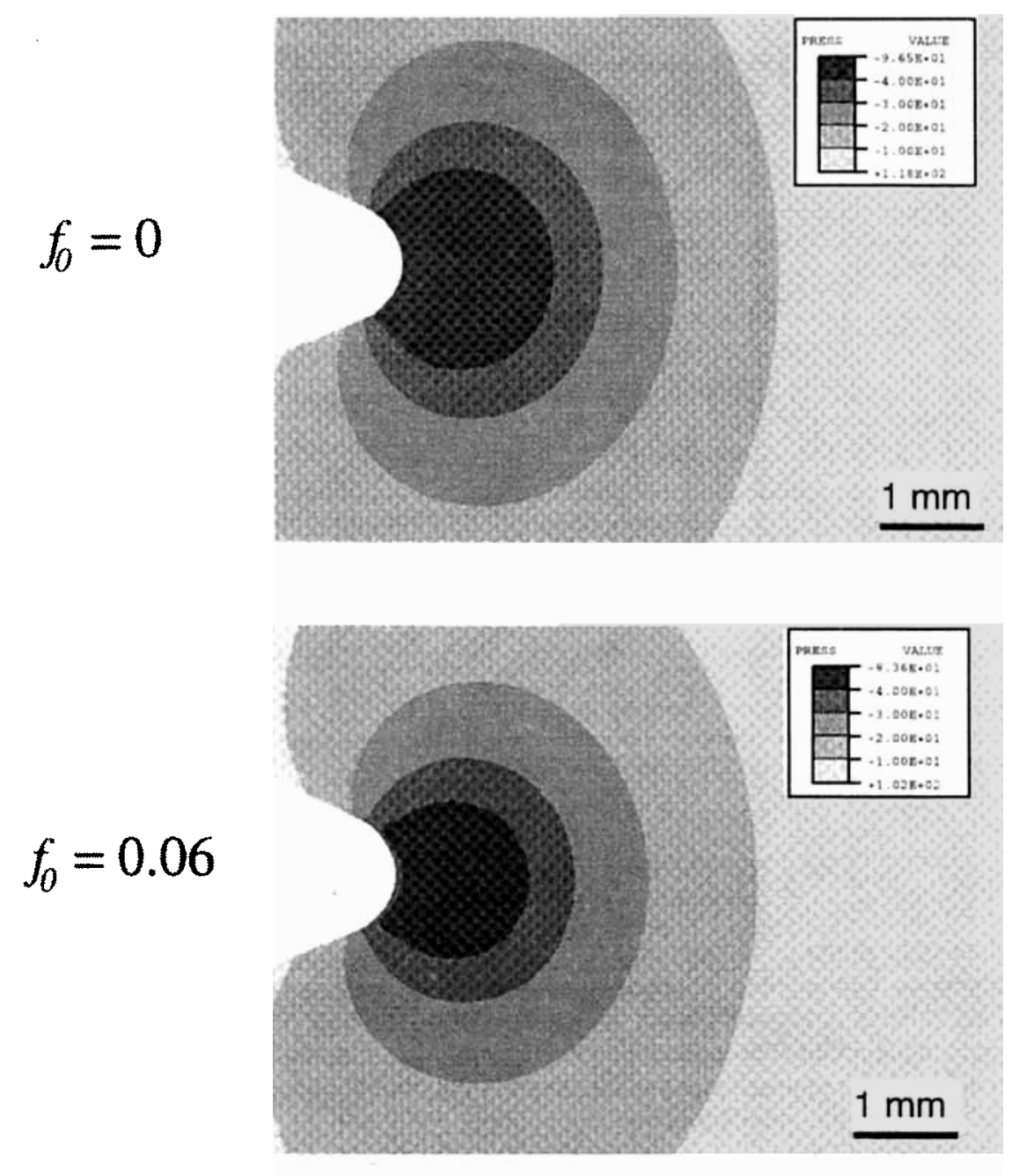

$f_{0}=0.12$

the distribution pattern when compared to that shown in Fig. 12. As for the case of the larger initial void volume fraction of $f_{0}=0.12$, shown in Fig. 14, while the peak stresses are reached at a certain distance away from the tip, the stresses decrease and then increase again as the radial distance to the notch tip decreases. It will be shown later that there is a substan- 


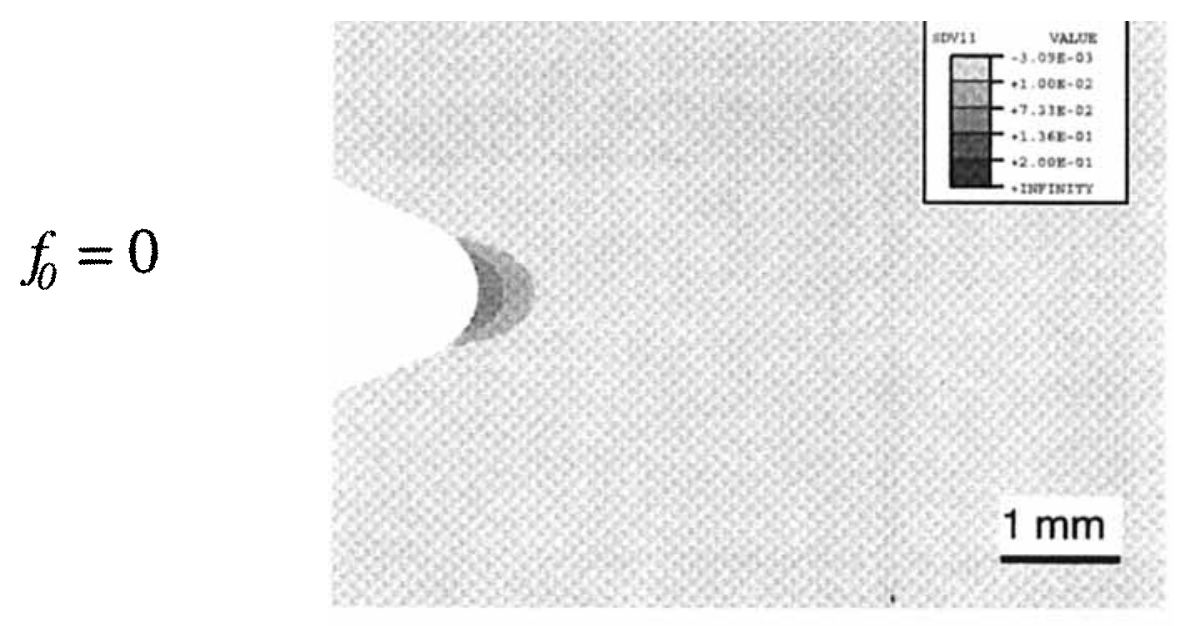

Fig. 16. Distributions of the equivalent tensile plastic strain near the notch tip for pressure-sensitive porous materials with the softenand $f_{0}=0,0.06$ and 0.12 . ing-hardening behavior, $\mu=0.13$,

$$
f_{0}=0.06
$$

$$
f_{0}=0.12
$$

The distributions of the macroscopic mean stress near the notch tip for pressure-sensitive porous materials with $\mu=0.13$ and the initial void volume fraction $f_{0}=0,0.06$ and 0.12 are shown in Fig. 15. We notice the change in the distribution from elliptical shape to
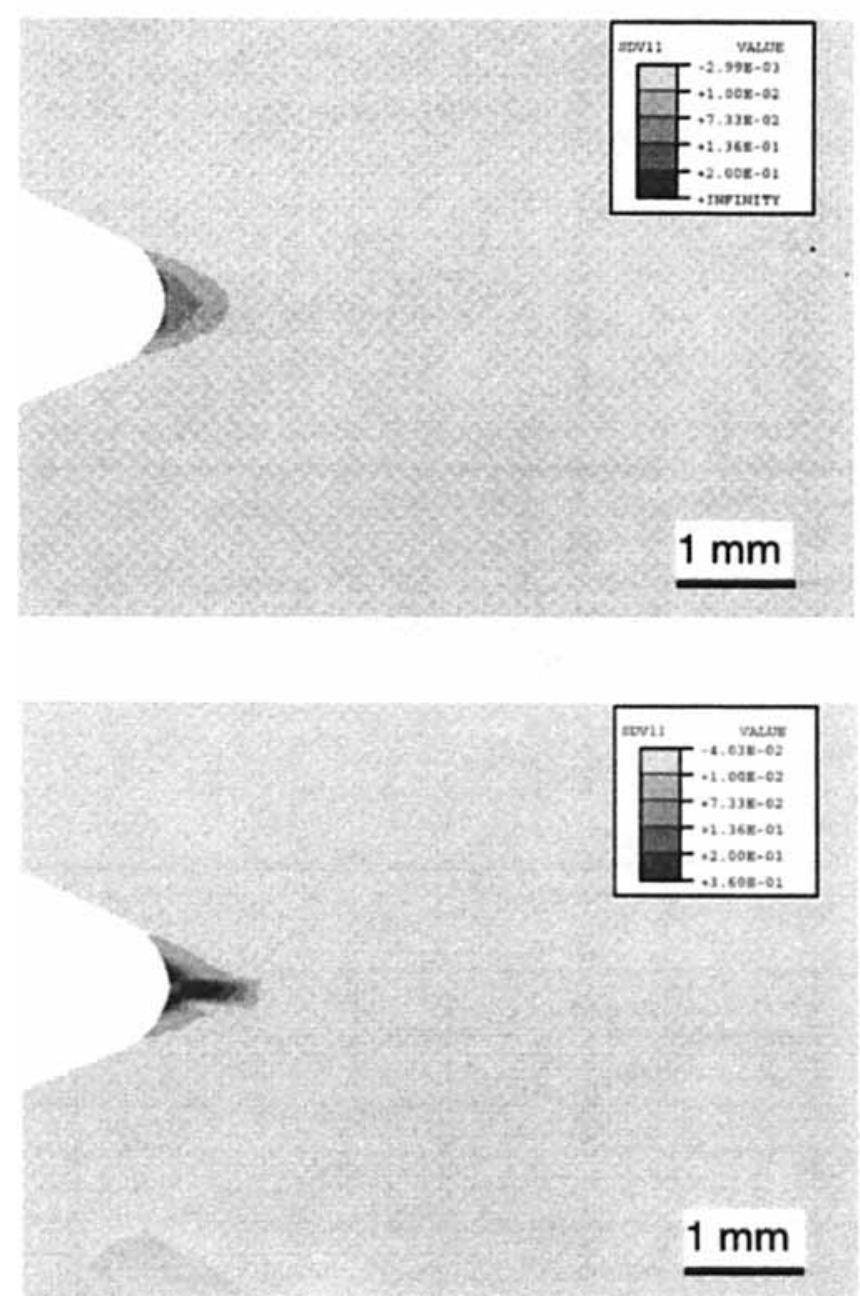

one closer to a circular shape for the mean stress equal to and larger than $20 \mathrm{MPa}$ as the void volume fraction increases. This is in agreement with the trend of the shapes of the observed cavitation zones reported in Yee et al. (13). For the larger value of the 


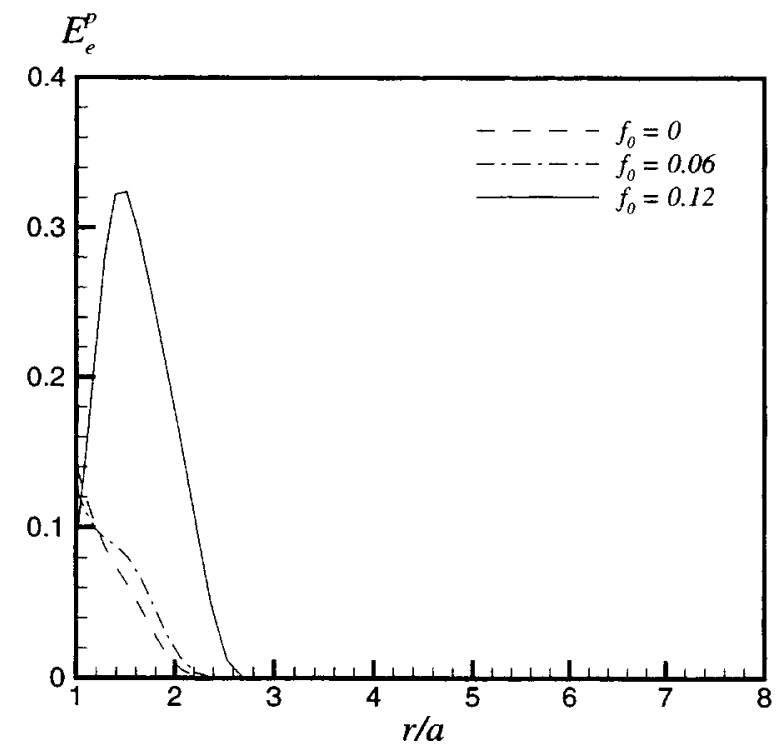

Fig. 17. Equivalent tensile plastic strains as functions of $\mathrm{r} / \mathrm{a}$ along the $y$ direction ahead of the notch tip for $\mu=0.13$ and $f_{0}=0,0.06$ and 0.12 .

void volume fraction $\left(f_{0}=0.12\right)$, the region near the notch tip with high mean stress (40 $\mathrm{MPa}$ or higher) becomes smaller. As seen in the bottom plot, there is a small circular region ahead of the notch tip, where the mean stress is quite low. This region is located between the notch tip and the high mean stress region (40 MPa or higher). This is the region where the void growth is substantial and the macroscopic effective plastic strain is higher, as will be shown later. The location of the low mean stress region is possibly corresponding to the crack initiation site as observed in (13).

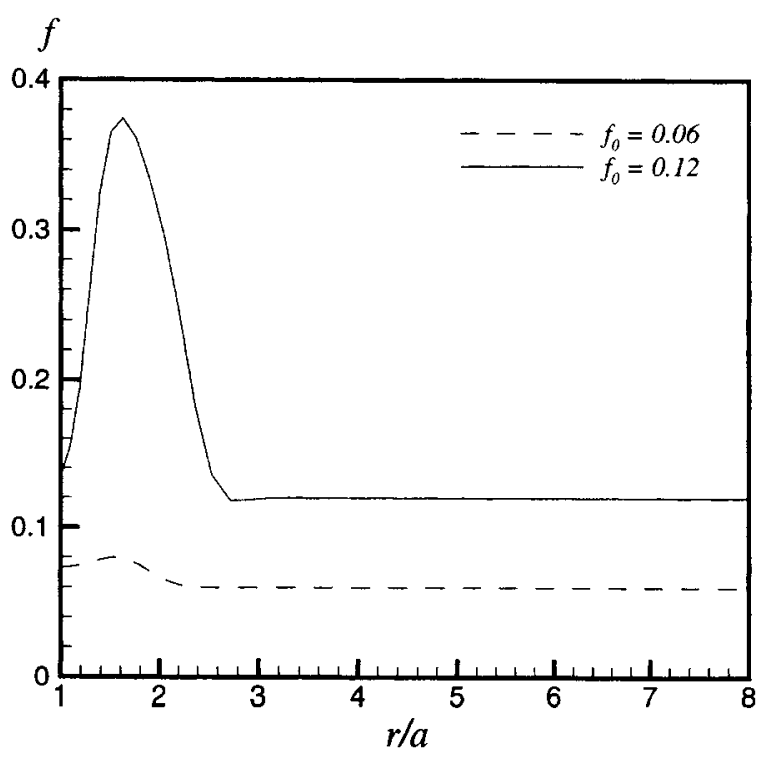

Fig. 18. Void volume fractions as functions of $r / a$ ahead of the notch tip for $\mu=0.13$ and $f_{0}=0.06$ and 0.12 .
The distributions of the equivalent tensile plastic strain $\left(E_{e}^{p}\right)$ for $f_{0}=0,0.06$ and 0.12 are shown in Fig. 16. The equivalent tensile plastic strain is defined in $E q$ 13. We notice that the zone defined by $E_{e}^{p} \geq 0.01$ grows gradually as $f_{0}$ increases. However, the intense shear yielding zone identified by $E_{e}^{p} \geq 0.2$ spreads some distance ahead of the notch tip only for $f_{0}=$ 0.12 , as seen in the bottom plot of Fig. 16. The shape of the intense shear yielding zone is somewhat similar to that seen in the experiments (13) although the extent of the region defined by $E_{e}^{p} \geq 0.01$ here is smaller. Note that the distribution of the equivalent tensile plastic strain is not exactly symmetrical for $f_{0}=0.12$ since the deformation is not symmetrical with respect to the notches for SDEDN specimens. For $f_{0}=0$ and 0.06 , the asymmetry of the distributions of the equivalent tensile plastic strains is not shown clearly as that shown in Fig. 15 for $f_{0}=0.12$.

Figure 17 shows the equivalent tensile plastic strains as functions of $r / a$ ahead of the notch tip for porous materials with $\mu=0.13$ and $f_{0}=0,0.06$ and 0.12 . As shown in the figure, the equivalent tensile plastic strain for the large initial void volume fraction case with $f_{0}=0.12$ is very large ahead of the notch tip. This large plastic strain is located near the low mean stress region ahead of the notch tip as discussed for Fig. 15.

Figure 18 shows the void volume fractions ahead of the notch tip for $\mu=0.13$ and $f_{0}=0.06$ and 0.12 . As shown in the figure, the void volume fractions far away from the notch tip in the elastic region stay at the original values. For the low initial void volume fraction $\left(f_{0}=0.06\right)$, the change in $f$ is very small in the plastic region near the notch tip. However, for the large initial void volume fraction case $\left(f_{0}=0.12\right)$, the void volume fraction increases dramatically due to void growth within the plastic region between the notch tip and the elastic-plastic boundary.

A comparison of the observations in Figs. 12-18 with the experimental results of Yee et al (13) shows that the overall cavitation zone observed in the subcritical damage zone near the notches on the tensile side of test specimens in the experiments is in agreement with the mean stress distribution presented here. The change in size and shape of the cavitation zone from elliptical to circular as the initial void volume fraction increases as discussed with regard to Fig. 15 can be seen in the experimental results shown in Fig. 8 of Yee et al. (13). Our computational results indicate that the intense shear zone is located directly ahead of the notch tip as shown in Fig. 16. However, the intense shear zones are smaller than the experimental results shown in Fig. 8 of Yee et al. (13).

The experimental observation of the relocation of crack initiation sites from some distance ahead of the notch for pure epoxy to locations closer to the notch tip for epoxies with higher rubber contents leads to the suggestion of the change of fracture mode from plane strain to plane stress proposed by Yee et al. (13). Note that the specimen is under plane strain 
conditions. Examining the stress and strain plots in Figs. 12, 13, and 17 for epoxies with no or small rubber content, we see that the maximum stresses are high and located at the elastic-plastic boundary. The plastic strains, however, increase gradually as the radial distance to the notch tip decreases, and reach the maximum at the free surface. The experimental results indicate that the crack initiation sites are far away from the tip (13). This indicates that the fracture mode may well be controlled by the stresses, and the nucleation sites are at the location of the maximum stresses.

On the other hand, when the rubber content increases, the maximum macroscopic stresses decrease, and the plastic strain becomes higher ahead of the notch tip for $f_{0}=0.12$. Examining the stress andstrain plots in Figs. 14,15,17, and 18 for the case of $f_{0}=0.12$ shows that the location of the maximum stresses is still farther away from the notch tip than that for pure epoxies, but very large plastic strains develop between the notch tip and the elastic-plastic boundary mainly due to the large initial void volume fraction and the growth of voids. The experimental results indicate that the nucleation sites are closer to the tip (13). This suggests that the fracture mode is now controlled by the plastic strains closer to the tip instead of the stresses since the maximum stresses ahead of the notch tip are not high enough to meet the stress-controlled fracture criterion. This explanation is consistent with the plane strain/plane stress argument proposed by Yee et al. (13) since we show that the large initial void volume fraction and the growth of voids decrease the macroscopic stress (or relieve the plane strain constraint) ahead of the tip, with the assumption that cavitation occurs early in the deformation.

\section{CONCLUSTONS}

In this work, we present notch-tip fields for pressure-sensitive non-porous and porous materials from our finite element analysis. The solutions for pressure-sensitive nonporous materials obtained here are in agreement with the analytical fully plastic solutions. The results for pressure-sensitive porous materials are in agreement with the experimental work of Yee et al. (13) for rubber-modified epoxies, where the cavitation zone is surrounding the notch tip and the intensive shear zone is located directly ahead of the notch tip. The computational results indicate that as the void volume fraction increases, the macroscopic stress becomes lower and the plastic strain is higher ahead of the notch tip. The location of the maximum plastic strain is closer to the notch tip when compared to the location of the maximum stresses for a large void volume fraction. This explains the change of fracture mode from being controlled by high mean stresses at the elastic-plastic boundary to being controlled by the plastic strain closer to the notch tip for epoxies with higher rubber contents, as indicated in the experiment by Yee et al. (13).

\section{ACKTOWLEDGMENT}

AA acknowledges the financial support of the Technical College of Riyadh, Riyadh, Saudi Arabia. JP acknowledges the initial support of this work by the NSF under grant number DMR—8708405.

\section{REFERENCES}

1. S. S. Sternstein and L. Ongchin, Am. Chem. Soc. Polym. Prep., 10, 1117 (1969).

2. S. Rabinowtz, I. M. Ward, and J. S. C. Perry, J. Mater. Sci, 5, 29 (1970).

3. W. A. Spitzig and O. Richmond, Polym. Eng. Sci., 18, 1129 (1979).

4. A. J. Kinloch and R. J. Young, Fracture Behavior of Polymers, Elsevier Applied Sciences, London (1983).

5. D. C. Drucker and W. Prager, Q. Appl. Math 10, 157 (1952).

6. D. C. Drucker, Metall. Trans. 4, 667 (1973).

7. F. J. McGarry, Proc. $R$. Soc. Lond, A319, 59 (1970).

8. J. N. Sultan, R. C. Laible and F. J. McGarry, App. Polym. Symposium, 16, 127 (1971).

9. J. N. Sultan, and F. J. McGarry, Polym. Eng. Sci, 13, 29 (1973).

10. A. F. Yee and R. A. Pearson, J. Mater Sci., 21, 2462 (1986).

11. R. A. Pearson and A. F. Yee, J. Mater. Sci, 21, 2475 (1986).

12. R. A. Pearson and A. F. Yee, J. Mater. Sci, 26, 3828 (1991).

13. A. F. Yee, D. Li and X. Li, J. Mater. Sci, 28, 6392 (1993).

14. V. Tvergaard, Euro. J. Mech A/Solids, 16, 5 (1997).

15. A. L. Gurson, J. Eng. Mater. Tech, 89, 2 (1977).

16. A. L. Gurson, Proceedings of the Fourth International Conference on Fracture, 2, p. 357, D. M. R. Taplin. ed.,Pergamon Press, Oxford, England (1977).

17. H.-Y. Jeong, PhD Thesis, The University of Michigan, Ann Arbor (1992).

18. H.-Y. Jeong and J. Pan, Int. J. Solids Struct., 32, 3669 (1995).

19. H.-Y. Jeong and J. Pan, Polym. Eng. Sci. 36, 2306 (1996).

20. W. J. Chang and J. Pan, "Cavitation instabilities in plastics and rubber-modified plastics," in press, Int. $J$. Fract., (1997).

21. A. Al-Abduljabbar and J. Pan, Polym. Eng. Sci., 38, 1031 (1998).

22. W. A. Spitzig, R. J. Sober, and O. Richmond, Act. Metal, 23, 885 (1975).

23. W. A. Spitzig, R. J. Sober, and O. Richmond, Metal. Trans.7A, 1703 (1976).

24. O. Richmond and W. A. Spitzig, International Union of Thoerertical and Applied Mechanics, 337 (1980).

25. I.-W. Chen, J. Am. Ceram. Soc., 74, 2564 (1991).

26 V. Tvergaard, Int. J. Fract, 17, 389 (1981).

27. V. Tvergaard, Int. J. Fract., 18, 237 (1982).

28 G. P. Tandon and G. J. Weng, J. Appl. Mech, 56, 126 (1988).

29. A. Al-Abduljabbar and J. Pan, "Effects of pressure sensitivity on the $\eta$ factor and the $J$ integral estimation for compact tension specimens," submitted for publication in J. Mater. Sci. (1997).

30. E. Van der Giessen, Euro. J. Mech. A/Solids, 16, 87 (1997).

31. A. N. Gent and P. B. Lindley, Proc. R. Soc., A248, 195 (1959). 